hep-ph/0204069

UT-02-19

April, 2002

\title{
(S)fermion Masses in Fat Brane Scenario
}

\author{
Naoyuki Haba ${ }^{a}$ and Nobuhito Maru ${ }^{b}$ \\ ${ }^{a}$ Faculty of Engneering, Mie University, Tsu, Mie, 514-8507, JAPAN \\ ${ }^{b}$ Department of Physics, University of Tokyo, Tokyo 113-0033, JAPAN
}

\begin{abstract}
We discuss the fermion mass hierarchy and the flavor mixings in the fat brane scenario of a five dimensional SUSY theory. Assuming that the matter fields lives in the bulk, their zero mode wave functions are Gaussians, and Higgs fields are localized on the brane, we find simple various types of the matter configurations generating the mass matrices consistent with experimental data. Sfermion mass spectrum is also discussed using the matter configurations found above. Which type of squark mass spectra (the degeneracy, the decoupling and the alignment) is realized depends on the relative locations of SUSY breaking brane and the brane where Higgs fields are localized.
\end{abstract}




\section{Introduction}

Utilizing extra dimensions has shed new insights into various phenomenological aspects of the physics in four dimensions. In particular, Arkani-Hamed and Schmaltz have proposed an interesting mechanism, which is refered to as "fat brane scenario", to generate small parameters naturally in the effective four dimensional theories from higher dimensional theories [1]. These small parameters are obtained by a small overlap of wave functions, even if the parameters in a fundamental theory are of order unity. This mechanism has been applied to various phenomenological issues so far, such as the fermion mass hierarchy [2, 3, 4, 5, 6, 7], the doublet-triplet splitting [8, 9, 10], and the sfermion mass generation [3], 11, 6, 12].

In this paper, we discuss the fermion mass hierarchy and the flavor mixings in the fat brane scenario. As mentioned above, some people has already considered this issue [2, 3, 4, 5, 6, 7], so it is necessary to clarify the difference between our setup and the others. The setup of authors [2, 4, 5] is as follows. A Higgs field lives in the bulk and its zero mode wave function is flat. The matter fields also live in the bulk and its zero mode wave functions are Gaussian and localized at different points in extra dimensions. The fermion mass hierarchy is generated by an overlap between the matter wave functions. The setup of authors [3, 7] is that the matter and Higgs live in the bulk and both zero mode wave functions are Gaussian and are localized at different points in extra dimensions. The fermion mass hierarchy is generated by an overlap between the matter and Higgs wave functions. On the other hand, our setup is slightly different from these two setups in that Higgs fields are localized on a brane. 7 As for the matter fields, our setup is the same as these two setups. The fermion mass hierarchy is determined by the values at a brane where Higgs fields are localized. We find various types of the matter configurations in a five dimensional supersymmetric (SUSY) theory, which yield the fermion mass matrices consistent with experimental data.

We also discuss the sfermion mass spectrum. Sfermion masses are generated by the overlap between the wave functions of matter filefds and the chiral superfileld with nonzero vacuum expectation value (VEV) of the F-component localized on the SUSY breaking brane. If SUSY breaking is located far from the matter fields, the sfermion mass spec-

\footnotetext{
${ }^{1}$ This setup has also been considered in Ref. [6]. The difference between their setup and ours is that they considered that the form of the zero mode wave functions of matter fields are exponential, but Gaussian in our case.
} 
trum is similar to the gaugino mediation scenario since the gaugino masses receive only the volume suppression but the sfermion masses receive additional exponential suppression. In this case, the degeneracy solution to SUSY flavor problem is expected as in the gaugino mediation. In Ref. [12], we have proposed that if SUSY breaking brane is located between the first and the second generations, the sfermion mass spectrum becomes the decoupling solution. But in [12], the flavor mixings were neglected in determining the matter configuration for simplicity. In this paper, this point is improved. We estimate the sfermion masses and the mixings and see whether the decoupling solution can be realized when we take into account both of the fermion masses and their flavor mixings. More interestingly, if SUSY breaking is located at the same point where Higgs fileds are localized, the sfermion masses are propotrtional to Yukawa couplings. In this case, the alignment solution to SUSY flavor problem is expected. Thus, which type of the sfermion spectra is realized depends on the relative location between a SUSY breaking brane and the brane where Higgs fileds are localized in our framework.

This paper is organized as follows. In the next section, we discuss the fermion mass hierarchy and the flavor mixings. We find various types of the matter configurations consistent with the experimental data in a five dimensional SUSY theory. In section 3, corresponding to the fermion masses found above, the sfermion mass spectrum is also discussed. Summary of our paper is given in the last section.

\section{Fermion masses and mixings}

In this section, we discuss the fermion masses and the flavor mixings in a five dimensional SUSY theory. Let us consider the up-type Yukawa coupling, for example,

$$
W=\delta(y) \int d y Q_{i}(x, y) \bar{U}_{j}(x, y) H_{u}(x)
$$

where $x$ denotes the coordinate of four dimensional Minkowski space-time, $y$ is a fifth spatial coordinate of five dimensions. $i, j$ are the generation indices. The order one

coefficient is implicit. $Q_{i}$ and $\bar{U}_{i}$ are the chiral superfield which transform as $(\mathbf{3}, \mathbf{2}, 1 / 6)$ and $\left(\mathbf{3}^{*}, \mathbf{1},-2 / 3\right)$ under the Standard Model $(\mathrm{SM})$ gauge groups, $S U(3)_{C} \times S U(2)_{L} \times U(1)_{Y}$. We assume here that the matter fields live in the bulk and Higgs fields are localized on a brane at $y=0$. On a thick wall, a zero mode wave function of the matter superfield with the left (or right)-handed chirality is localized, and the matter superfield with an opposite 
chirality is not localized. Integrating out the fifth dimensional degrees of freedom, we obtain the effective Yukawa coupling in four dimensions at the compactification scale as,

$$
\left(y_{\mathrm{eff}}\right)_{i j} \simeq \exp \left[-a^{2}\left(y_{Q_{i}}^{2}+y_{\bar{U}_{j}}^{2}\right)\right]
$$

where we assume the form of the zero mode wave function of the matter superfields to be Gaussian such as $\exp \left[-a^{2}\left(y-y_{\Phi_{i}}\right)^{2}\right]$, where $M_{*}$ is the five dimensional Planck scale and $a$ is the width of the matter zero mode wave functions. $y_{\Phi_{i}}$ is the coordinate where the matter superfield $\Phi_{i}\left(=Q_{i}, \bar{U}_{i}, \bar{D}_{i}, \ldots\right)$ is localized. As is clear from (2) , the information of Yukawa hierarchy is interpreted as the configuration of the matter fields in the extra dimensions.

In the following subsections, we consider various types of fermion mass matrices, which well describe the fermion mass hierarchies and the flavor mixings, and discuss whether these mass matrices can be realized from the fat brane point of view. Note that some of these mass matrices have been discussed in the context of five or six dimensional SUSY orbifold Grand Unified Theories (GUT) [13, 14.

\section{1 "Anarchy type" fermion mass matrices}

At first, let us consider the following matter configuration in the fifth dimensional coordinate.

$$
\begin{aligned}
& q_{1}^{2} \simeq-2 \ln \epsilon, \quad u_{1}^{2} \simeq-2 \ln \epsilon, \quad d_{1}^{2} \simeq-\ln \epsilon, \\
& q_{2}^{2} \simeq-\ln \epsilon, \quad u_{2}^{2} \simeq-\ln \epsilon, \quad d_{2}^{2} \simeq-\ln \epsilon, \\
& q_{3}^{2} \simeq 0, \quad u_{3}^{3} \simeq 0, \quad d_{3}^{2} \simeq-\ln \epsilon, \\
& l_{1}^{2} \simeq 0, \quad e_{1}^{2} \simeq-3 \ln \epsilon, \quad n_{1}^{2} \simeq 0, \\
& l_{2}^{2} \simeq 0, \quad e_{2}^{2} \simeq-2 \ln \epsilon, \quad n_{2}^{2} \simeq 0, \\
& l_{3}^{2} \simeq 0, \quad e_{3}^{2} \simeq-\ln \epsilon, \quad n_{3}^{2} \simeq 0 \text {, }
\end{aligned}
$$

where $q_{i}$ means the coordinate in units of $a^{-1}$ where $Q_{i}$ is localized, for instance. The configuration (3) generates the following mass matrices for up, down quark sectors and the charged lepton sector:

$$
m_{u} \simeq\left(\begin{array}{ccc}
\epsilon^{4} & \epsilon^{3} & \epsilon^{2} \\
\epsilon^{3} & \epsilon^{2} & \epsilon \\
\epsilon^{2} & \epsilon & 1
\end{array}\right)\left\langle H_{u}\right\rangle, m_{d} \simeq \epsilon\left(\begin{array}{ccc}
\epsilon^{2} & \epsilon^{2} & \epsilon^{2} \\
\epsilon & \epsilon & \epsilon \\
1 & 1 & 1
\end{array}\right)\left\langle H_{d}\right\rangle, m_{l} \simeq \epsilon\left(\begin{array}{ccc}
\epsilon^{2} & \epsilon & 1 \\
\epsilon^{2} & \epsilon & 1 \\
\epsilon^{2} & \epsilon & 1
\end{array}\right)\left\langle H_{d}\right\rangle,
$$

\footnotetext{
${ }^{2}$ For readers interested in the localization mechanism of the chiral superfields, see an appendix in Ref. [3].
} 
where $\epsilon \simeq \lambda^{2}$, and $\lambda$ is the Cabibbo angle, $\lambda \simeq 0.2$. The above mass matrices suggest the small $\tan \beta$, and generate the fermion mass hierarchies,

$$
\begin{aligned}
& m_{t}: m_{c}: m_{u} \simeq 1: \epsilon^{2}: \epsilon^{4} \\
& m_{b}: m_{s}: m_{d} \simeq m_{\tau}: m_{\mu}: m_{e} \simeq 1: \epsilon: \epsilon^{2} .
\end{aligned}
$$

The mass matrices of the left-handed neutrino and the right-handed neutrino sectors are

$$
m_{\nu}^{D} \simeq\left(\begin{array}{ccc}
1 & 1 & 1 \\
1 & 1 & 1 \\
1 & 1 & 1
\end{array}\right)\left\langle H_{d}\right\rangle, m_{N} \simeq\left(\begin{array}{ccc}
1 & 1 & 1 \\
1 & 1 & 1 \\
1 & 1 & 1
\end{array}\right) M_{R}
$$

where $M_{R}$ is around $10^{15-16} \mathrm{GeV}$. It is because the Majorana mass term in our model is assumed to be generated by a nonzero VEV of the singlet $S$ field 5 localized on the brane at $y=0$ with the term,

$$
\delta(y) \frac{1}{2 M_{*}} S(x) N_{i}(x, y) N_{j}(x, y)
$$

Integrating out the fifth dimensional degrees of freedom and taking the VEV of $S$ to be $M_{*}$, we obtain $M_{R} \simeq \sqrt{\frac{2}{\pi}} a^{-1} \simeq 0.8 \times a^{-1}$. In the fat brane scenario, $a^{-1}$ is at most $L_{c}^{-1} \simeq 10^{16} \mathrm{GeV}$, so $M_{R} \simeq 8.0 \times 10^{15} \mathrm{GeV}$. This is somewhat large compared to $M_{R} \simeq 10^{14} \mathrm{GeV}$ which is the suitable magnitude for the neutrino oscillation experiments, but taking into account $O(1)$ coefficients in neutrino mass matrices, the value, $10^{15} \mathrm{GeV}$, is sufficient. From these matrices, the mass matrix of three light neutrinos $m_{\nu}^{(l)}$ via see-saw mechanism [15] is obtained as

$$
m_{\nu}^{(l)} \simeq \frac{m_{\nu}^{D}\left(m_{\nu}^{D}\right)^{t}}{m_{N}} \simeq\left(\begin{array}{lll}
1 & 1 & 1 \\
1 & 1 & 1 \\
1 & 1 & 1
\end{array}\right) \frac{\left\langle H_{u}\right\rangle^{2}}{M_{R}}
$$

By diagonalizing these matrices, the CKM [16] and the MNS [17] mixing matrices are obtained,

$$
V_{\mathrm{CKM}} \simeq\left(\begin{array}{ccc}
1 & \epsilon & \epsilon^{2} \\
\epsilon & 1 & \epsilon \\
\epsilon^{2} & \epsilon & 1
\end{array}\right), V_{\mathrm{MNS}} \simeq\left(\begin{array}{ccc}
1 & 1 & 1 \\
1 & 1 & 1 \\
1 & 1 & 1
\end{array}\right)
$$

They can naturally explain why the flavor mixing in the quark sector is small while the flavor mixing in the lepton sector is large [18, 19, 20]. The above mass matrices and flavor

\footnotetext{
3 The field $S$ have the lepton number in two units. Thus its VEV might derive a massless Majoron, naively. To discuss a Majoron is beyond the scope of this paper. We simply asuume here, for example, the lepton number is explicitly broken in some sectors and avoid a massless Majoron.
} 
mixings are roughly consistent with the experimental data, and explicit values of $O(1)$ coefficients of mass matrices can really induce the suitable magnitudes of fermion masses and flavor mixing angles [18]. The random coefficients have suggested the probabilities of fermion masses and flavor mixings [20]21]. If we would like to obtain the suitably large (small) Cabibbo angle $\left(U_{e 3}\right)$, and small $m_{d, e}$, of order the power of $\lambda$, the matter configuration should be modified. We will show two examples of the improved fermion mass matrices in the following two subsections.

The configuration corresponding to the large $\tan \beta$ case is also realized by changing the locations of $\bar{D}_{1,2,3}$ and $\bar{E}_{1,2,3}$ in (3) as,

$$
\begin{array}{lll}
q_{1}^{2} \simeq-2 \ln \epsilon, & u_{1}^{2} \simeq-2 \ln \epsilon, & d_{1}^{2} \simeq 0 \\
q_{2}^{2} \simeq-\ln \epsilon, & u_{2}^{2} \simeq-\ln \epsilon, & d_{2}^{2} \simeq 0 \\
q_{3}^{2} \simeq 0, & u_{3}^{3} \simeq 0, & d_{3}^{2} \simeq 0 \\
l_{1}^{2} \simeq 0, & e_{1}^{2} \simeq-2 \ln \epsilon, & n_{1}^{2} \simeq 0 \\
l_{2}^{2} \simeq 0, & e_{2}^{2} \simeq-\ln \epsilon, & n_{2}^{2} \simeq 0 \\
l_{3}^{2} \simeq 0, & e_{3}^{2} \simeq 0, & n_{3}^{2} \simeq 0
\end{array}
$$

This configuration is more interesting than the previous configuration since the localization patterns for $Q_{i}, \bar{U}_{i}, \bar{E}_{i}$ and $\bar{D}_{i}, L_{i}$ are the same as for the generation index. This implies that this case can be embeded into the SU(5) GUT. The mass matrices for up, down quark sectors and the charged lepton sector are given by

$$
m_{u} \simeq\left(\begin{array}{ccc}
\epsilon^{4} & \epsilon^{3} & \epsilon^{2} \\
\epsilon^{3} & \epsilon^{2} & \epsilon \\
\epsilon^{2} & \epsilon & 1
\end{array}\right)\left\langle H_{u}\right\rangle, m_{d} \simeq\left(\begin{array}{ccc}
\epsilon^{2} & \epsilon^{2} & \epsilon^{2} \\
\epsilon & \epsilon & \epsilon \\
1 & 1 & 1
\end{array}\right)\left\langle H_{d}\right\rangle, m_{l} \simeq\left(\begin{array}{ccc}
\epsilon^{2} & \epsilon & 1 \\
\epsilon^{2} & \epsilon & 1 \\
\epsilon^{2} & \epsilon & 1
\end{array}\right)\left\langle H_{d}\right\rangle .
$$

Since the difference between the matrices (四) and (12) only exists in an overall factor, the arguments for the fermion mass hierarchies and the flavor mixings are the same as those of the small $\tan \beta$ case.

Here we would like to stress the importance of the configurations found above. They are simpler than other configurations in the literatures [2, 3, 4, 5, 6, 7] in the following points:

1. The configurations are obtained in a five dimensional theory,

2. The width of the wave functions is common to all the matter fields,

3. The distribution of the matter superfields is very simple. 


\subsection{Improved mass matrices I}

From the view point of order estimation, the mass matrices discussed in the previous subsection gives rather small Cabibbo angles and rather large $U_{e 3}$. As discussed in Ref. [14], there are two improvements of the "Anarchy type" of fermion mass matrices. In our framework, these improvements can be easily done by changing the locations of $\bar{D}_{1}, L_{1}$ and $\bar{N}_{1}$ in (3). Let us show the first example of the improvements here. The configuration is given by

$$
\begin{aligned}
& q_{1}^{2} \simeq-2 \ln \epsilon, \quad u_{1}^{2} \simeq-2 \ln \epsilon, \quad d_{1}^{2} \simeq-2 \ln \epsilon, \\
& q_{2}^{2} \simeq-\ln \epsilon, \quad u_{2}^{2} \simeq-\ln \epsilon, \quad d_{2}^{2} \simeq-\ln \epsilon, \\
& q_{3}^{2} \simeq 0, \quad u_{3}^{3} \simeq 0, \quad d_{3}^{2} \simeq-\ln \epsilon, \\
& l_{1}^{2} \simeq-\ln \epsilon, \quad e_{1}^{2} \simeq-3 \ln \epsilon, \quad n_{1}^{2} \simeq-\ln \epsilon, \\
& l_{2}^{2} \simeq 0, \quad e_{2}^{2} \simeq-2 \ln \epsilon, \quad n_{2}^{2} \simeq 0, \\
& l_{3}^{2} \simeq 0, \quad e_{3}^{2} \simeq-\ln \epsilon, \quad n_{3}^{2} \simeq 0 .
\end{aligned}
$$

This configuration is simple enough as that of "Anarchy type". The mass matrices for up, down quark sectors and the charged lepton sector from this configuration are

$$
m_{u} \simeq\left(\begin{array}{ccc}
\epsilon^{4} & \epsilon^{3} & \epsilon^{2} \\
\epsilon^{3} & \epsilon^{2} & \epsilon \\
\epsilon^{2} & \epsilon & 1
\end{array}\right)\left\langle H_{u}\right\rangle, m_{d} \simeq \epsilon\left(\begin{array}{ccc}
\epsilon^{3} & \epsilon^{2} & \epsilon^{2} \\
\epsilon^{2} & \epsilon & \epsilon \\
\epsilon & 1 & 1
\end{array}\right)\left\langle H_{d}\right\rangle, m_{l} \simeq \epsilon\left(\begin{array}{ccc}
\epsilon^{3} & \epsilon^{2} & \epsilon \\
\epsilon^{2} & \epsilon & 1 \\
\epsilon^{2} & \epsilon & 1
\end{array}\right)\left\langle H_{d}\right\rangle
$$

The mass hierarchies in the down quark sector and the charged lepton sector are modified as

$$
\begin{aligned}
& m_{t}: m_{c}: m_{u} \simeq 1: \epsilon^{2}: \epsilon^{4} \\
& m_{b}: m_{s}: m_{d} \simeq m_{\tau}: m_{\mu}: m_{e} \simeq 1: \epsilon: \epsilon^{3}
\end{aligned}
$$

This suggests the suitable order of magnitudes of $m_{e}$ and $m_{d}$.

The mass matrices of the left-handed neutrino sector and the right-handed neutrino sector are given by

$$
m_{\nu}^{D} \simeq\left(\begin{array}{ccc}
\epsilon^{2} & \epsilon & \epsilon \\
\epsilon & 1 & 1 \\
\epsilon & 1 & 1
\end{array}\right)\left\langle H_{d}\right\rangle, m_{N} \simeq\left(\begin{array}{ccc}
\epsilon^{2} & \epsilon & \epsilon \\
\epsilon & 1 & 1 \\
\epsilon & 1 & 1
\end{array}\right) M_{R}
$$

The mass matrix of three light neutrinos $m_{\nu}^{(l)}$ via see-saw mechanism is given by

$$
m_{\nu}^{(l)} \simeq \frac{m_{\nu}^{D}\left(m_{\nu}^{D}\right)^{t}}{m_{N}} \simeq\left(\begin{array}{ccc}
\epsilon^{2} & \epsilon & \epsilon \\
\epsilon & 1 & 1 \\
\epsilon & 1 & 1
\end{array}\right) \frac{\left\langle H_{u}\right\rangle^{2}}{M_{R}}
$$


Here we assume the rank of $2 \times 2$ submatrix for the second and the third generations in $m_{\nu}^{(l)}$ is reduced. Otherwise, the large mixing between the first and the second generations cannot be realized. When this rank reduction of the submatrix is assumed in (17), the CKM and the MNS matrices are obtained as

$$
V_{\mathrm{CKM}} \simeq\left(\begin{array}{ccc}
1 & \epsilon & \epsilon^{2} \\
\epsilon & 1 & \epsilon \\
\epsilon^{2} & \epsilon & 1
\end{array}\right), V_{\mathrm{MNS}} \simeq\left(\begin{array}{ccc}
1 / \sqrt{2} & 1 / \sqrt{2} & \epsilon \\
1 / 2 & -1 / 2 & 1 / \sqrt{2} \\
-1 / 2 & 1 / 2 & 1 / \sqrt{2}
\end{array}\right) .
$$

This type of the MNS matrix is so-called bi-maximal one.

The configuration corresponding to the large $\tan \beta$ case is easily obtained by changing the locations of $\bar{D}_{1,2,3}$ and $\bar{E}_{1,2,3}$ in (13) as

$$
\begin{array}{lll}
q_{1}^{2} \simeq-2 \ln \epsilon, & u_{1}^{2} \simeq-2 \ln \epsilon, & d_{1}^{2} \simeq-\ln \epsilon, \\
q_{2}^{2} \simeq-\ln \epsilon, & u_{2}^{2} \simeq-\ln \epsilon, & d_{2}^{2} \simeq 0, \\
q_{3}^{2} \simeq 0, & u_{3}^{3} \simeq 0, & d_{3}^{2} \simeq 0, \\
l_{1}^{2} \simeq-\ln \epsilon, & e_{1}^{2} \simeq-2 \ln \epsilon, & n_{1}^{2} \simeq-\ln \epsilon, \\
l_{2}^{2} \simeq 0, & e_{2}^{2} \simeq-\ln \epsilon, & n_{2}^{2} \simeq 0, \\
l_{3}^{2} \simeq 0, & e_{3}^{2} \simeq 0, & n_{3}^{2} \simeq 0
\end{array}
$$

The corresponding mass matrices of the up and down quark sectors and the charged lepton sector are

$$
m_{u} \simeq\left(\begin{array}{ccc}
\epsilon^{4} & \epsilon^{3} & \epsilon^{2} \\
\epsilon^{3} & \epsilon^{2} & \epsilon \\
\epsilon^{2} & \epsilon & 1
\end{array}\right)\left\langle H_{u}\right\rangle, m_{d} \simeq\left(\begin{array}{ccc}
\epsilon^{3} & \epsilon^{2} & \epsilon^{2} \\
\epsilon^{2} & \epsilon & \epsilon \\
\epsilon & 1 & 1
\end{array}\right)\left\langle H_{d}\right\rangle, m_{l} \simeq\left(\begin{array}{ccc}
\epsilon^{3} & \epsilon^{2} & \epsilon \\
\epsilon^{2} & \epsilon & 1 \\
\epsilon^{2} & \epsilon & 1
\end{array}\right)\left\langle H_{d}\right\rangle .
$$

This case can be embeded into the $\mathrm{SU}(5)$ GUT. The results for the fermion mass hierarchies and the flavor mixings are the same as those of the small $\tan \beta$ case.

\subsection{Improved mass matrices II}

The second example of the improvements shows the changes of the locations of $\bar{D}_{1,2,3}, L_{1,2,3}$ and $\bar{N}_{1,2,3}$ in (3). The matter configuration is

$$
\begin{aligned}
& q_{1}^{2} \simeq-2 \ln \epsilon, \quad u_{1}^{2} \simeq-2 \ln \epsilon, \quad d_{1}^{2} \simeq-3 \ln \epsilon, \\
& q_{2}^{2} \simeq-\ln \epsilon, \quad u_{2}^{2} \simeq-\ln \epsilon, \quad d_{2}^{2} \simeq-2 \ln \epsilon, \\
& q_{3}^{2} \simeq 0, \quad u_{3}^{3} \simeq 0, \quad d_{3}^{2} \simeq-2 \ln \epsilon, \\
& l_{1}^{2} \simeq-2 \ln \epsilon, \quad e_{1}^{2} \simeq-3 \ln \epsilon, \quad n_{1}^{2} \simeq-2 \ln \epsilon, \\
& l_{2}^{2} \simeq-\ln \epsilon, \quad e_{2}^{2} \simeq-2 \ln \epsilon, \quad n_{2}^{2} \simeq-\ln \epsilon, \\
& l_{3}^{2} \simeq-\ln \epsilon, \quad e_{3}^{2} \simeq-\ln \epsilon, \quad n_{3}^{2} \simeq-\ln \epsilon .
\end{aligned}
$$

The resulting mass matrices for up, down quark sectors and the charged lepton sector are

$$
m_{u} \simeq\left(\begin{array}{ccc}
\epsilon^{4} & \epsilon^{3} & \epsilon^{2} \\
\epsilon^{3} & \epsilon^{2} & \epsilon \\
\epsilon^{2} & \epsilon & 1
\end{array}\right)\left\langle H_{u}\right\rangle, m_{d} \simeq \epsilon^{2}\left(\begin{array}{ccc}
\epsilon^{3} & \epsilon^{2} & \epsilon^{2} \\
\epsilon^{2} & \epsilon & \epsilon \\
\epsilon & 1 & 1
\end{array}\right)\left\langle H_{d}\right\rangle, m_{l} \simeq \epsilon^{2}\left(\begin{array}{ccc}
\epsilon^{3} & \epsilon^{2} & \epsilon \\
\epsilon^{2} & \epsilon & 1 \\
\epsilon^{2} & \epsilon & 1
\end{array}\right)\left\langle H_{d}\right\rangle,
$$


and those of the left-handed neutrino sector and the right-handed neutrino sector are given by

$$
m_{\nu}^{D} \simeq \epsilon^{2}\left(\begin{array}{ccc}
\epsilon^{2} & \epsilon & \epsilon \\
\epsilon & 1 & 1 \\
\epsilon & 1 & 1
\end{array}\right)\left\langle H_{d}\right\rangle, m_{N} \simeq \epsilon^{2}\left(\begin{array}{ccc}
\epsilon^{2} & \epsilon & \epsilon \\
\epsilon & 1 & 1 \\
\epsilon & 1 & 1
\end{array}\right) M_{R}
$$

Then, the mass matrix of three light neutrinos $m_{\nu}^{(l)}$ via the see-saw mechanism is given by

$$
m_{\nu}^{(l)} \simeq \frac{m_{\nu}^{D}\left(m_{\nu}^{D}\right)^{t}}{m_{N}} \simeq \epsilon^{2}\left(\begin{array}{ccc}
\epsilon^{2} & \epsilon & \epsilon \\
\epsilon & 1 & 1 \\
\epsilon & 1 & 1
\end{array}\right) \frac{\left\langle H_{u}\right\rangle^{2}}{M_{R}}
$$

Above mass matrices are the same as those of improvement I except for the overall factors. The fermion mass hierarchies of quarks and leptons are the same as those of improvement I. The flavor mixing matrices, $V_{\mathrm{CKM}}$ and $V_{\mathrm{MNS}}$, are also the same as those of the improvement I. The difference between improvement I and II exists in the value of $\tan \beta$. Compairing to the improvement I, the small $\tan \beta$ is prefer in the improvement II.

We can also find another configuration by changing the locations of $\bar{D}_{1,2,3}$ and $\bar{E}_{1,2,3}$ in $(23)$,

$$
\begin{aligned}
& q_{1}^{2} \simeq-2 \ln \epsilon, \quad u_{1}^{2} \simeq-2 \ln \epsilon, \quad d_{1}^{2} \simeq-2 \ln \epsilon, \\
& q_{2}^{2} \simeq-\ln \epsilon, \quad u_{2}^{2} \simeq-\ln \epsilon, \quad d_{2}^{2} \simeq-\ln \epsilon, \\
& q_{3}^{2} \simeq 0, \quad u_{3}^{3} \simeq 0, \quad d_{3}^{2} \simeq-\ln \epsilon, \\
& l_{1}^{2} \simeq-\ln \epsilon, \quad e_{1}^{2} \simeq-3 \ln \epsilon, \quad n_{1}^{2} \simeq-\ln \epsilon, \\
& l_{2}^{2} \simeq 0, \quad e_{2}^{2} \simeq-2 \ln \epsilon, \quad n_{2}^{2} \simeq 0, \\
& l_{3}^{2} \simeq 0, \quad e_{3}^{2} \simeq-\ln \epsilon, \quad n_{3}^{2} \simeq 0 .
\end{aligned}
$$

This configuration is nothing but that of the improvement I (13).

\section{Sfermion Masses}

In this section, we would like to discuss the sfermion mass spectrum, especially the squark mass spectrum. In extra dimensions, the sfermion masses correlate with the fermion masses by introducing SUSY breaking brane because the sfermion masses are determined by the overlap of wave functions of the matter fields and the chiral superfields with nonvanishing F-term VEV on SUSY breaking brane. Since we have determined the matter configuration consistent with experimental data in the previous section, the sfermion mass spectrum can be calculated and predicted. 
The gaugino masses are generated at tree level since we assume that the gauge supermultiplets live in a thick wall throughout this paper,

$$
\delta(y-L) \int d^{2} \theta \frac{X(x)}{M_{*}^{2}} W^{\alpha}(x, y) W_{\alpha}(x, y) \Rightarrow M_{\lambda}=\frac{F}{M_{*}^{2} L_{c}},
$$

where $W_{\alpha}$ is the field strength tensor superfield and $L_{c}$ is the width of the thick wall which should be considered as the compactification length in our framework. The gaugino masses should be around $100 \mathrm{GeV}$, so we obtain

$$
\frac{F}{M_{*}} \simeq 100\left(M_{*} L_{c}\right)
$$

In our previous paper, we have proposed the mechanism to generate the sfermion masses in the fat brane scenario [12]. Let us briefly review the scenario. A brane, we call "SUSY breaking brane", is introduced at $y=L$, where the chiral superfields $X$ with nonvanishing F-term $\operatorname{VEV}\left(X=\theta^{2} F\right)$ is assumed to be localized. The extra vector-like superfields $\Phi^{\prime}, \bar{\Phi}^{\prime}$ with mass $M<M_{*}$ are also introduced and assumed to be localized on a SUSY breaking brane. We consider here the following superpotential,

$$
\begin{aligned}
W & =\delta(y-L) \int d y\left[\frac{\lambda}{\sqrt{M_{*}}} X(x) \Phi_{i}(x, y) \bar{\Phi}^{\prime}(x)+M \Phi(x)^{\prime} \bar{\Phi}^{\prime}(x)\right] \\
& =\frac{\lambda}{\sqrt{M_{*} a}} \exp \left[-a^{2}\left(L-y_{\Phi_{i}}\right)^{2}\right] X(x) \Phi_{i}(x) \bar{\Phi}^{\prime}(x)+M \Phi^{\prime}(x) \bar{\Phi}^{\prime}(x),
\end{aligned}
$$

where $\lambda$ is a dimensionless constant of order unity. Below the scale $M$, we can integrate out the massive superfields $\Phi^{\prime}$ and $\bar{\Phi}^{\prime}$, then the superpotential vanishes and the effective Kähler potential receives the correction at tree level,

$$
\delta K=\frac{1}{\sqrt{M_{*} a}} \frac{1}{M^{2}} \exp \left[-a^{2}\left\{\left(L-y_{\Phi_{i}}\right)^{2}+\left(L-y_{\Phi_{j}}\right)^{2}\right\}\right] X^{\dagger} X \Phi_{i}^{\dagger} \Phi_{j}
$$

The sfermion masses coming from (31) at the compactification scale are

$$
\tilde{m}_{i j}^{2} \simeq \frac{1}{\sqrt{M_{*} a}} \exp \left[-a^{2}\left\{\left(L-y_{\Phi_{i}}\right)^{2}+\left(L-y_{\Phi_{j}}\right)^{2}\right\}\right] \frac{|F|^{2}}{M^{2}}
$$

It is crucial that the scale suppressing the Kähler potential is replaced with $M<M_{*}$ not so as to be negligibly small.f Note that $F<M^{2}$ is assumed in this argument and also the overall sign of the Kähler potential is assumed to be positive.

\footnotetext{
${ }^{4}$ Without introducing the extra vector-like superfields, the sfermion masses are negligibly small due to the exponential suppression [12].
} 
Before discussing the sfermion masses, we would like to mention A-terms in our scenario. In our scenario, A-terms are induced, for instance, from the term

$$
\int d^{2} \theta \frac{X}{M_{*}^{2}} Q_{i} \bar{U}_{j} H_{u}
$$

By integrating out the fifth dimensional degrees of freedom, it turns out immediately that A-terms are vanishing unless the SUSY breaking brane is located at the same point where Higgs fields are localized. Nonvanishing A-parameters are expressed by

$$
A=\frac{F}{M_{*}^{2} a}\left(y_{\mathrm{eff}}\right)_{i j}
$$

It is remarkable that in our framework A-parameters are necessarily proportional to Yukawa coupling constants.

If SUSY breaking brane is located apart from the brane where Higgs fields are localized, the sfermion masses (32) are negligibly small compared to the gaugino masses (27) since the sfermion masses receive the additional exponential suppression factor. This boundary condition is similar to the gaugino mediation scenario [24]. Therefore, the degeneracy solution to SUSY flavor problem is expected as in the gaugino mediation scenario.

In [12], we have proposed the decoupling solution in the fat brane scenatio. Now, we shall discuss this scenario. If a pair of vector-like superfields are introduced for each matter chiral superfield $Q_{i}, \bar{U}_{i}, \bar{D}_{i} \ldots$, we obtain the Kähler potential with no flavor mixings. We consider here this case, for simplicity. Sfermion masses generating from the Kähler potential with no flavor mixings are

$$
\tilde{m}_{i}^{2}=\left(\exp \left[-a^{2}\left(L-y_{\Phi_{i}}\right)^{2}\right]\right)^{2} \frac{1}{M_{*} a} \frac{|F|^{2}}{M^{2}}
$$

Now, we apply this mechanism to three types of the matter configuration found in the previous section and discuss whether the decoupling solution can be realized or not. Let us begin with the "Anarchy" model. Since the decoupling solution requires that the masses of the first and the second generations should be of $\mathcal{O}(10 \mathrm{TeV})$ and that of the third generation be around $\mathcal{O}(100 \mathrm{GeV})$ for naturalness, namely,

$$
\begin{aligned}
& \tilde{m}_{Q_{3}} \simeq \tilde{m}_{\bar{U}_{3}} \simeq 100 \mathrm{GeV} \Rightarrow \frac{1}{\sqrt{M_{*} a}} \frac{F}{M} \exp \left[-(a L)^{2}\right] \simeq 100 \mathrm{GeV} \\
& \tilde{m}_{Q_{1}} \simeq \tilde{m}_{\bar{U}_{1}} \simeq 10 \mathrm{TeV} \Rightarrow \frac{1}{\sqrt{M_{*} a}} \frac{F}{M} \exp \left[-(a L-\sqrt{-2 \ln \epsilon})^{2}\right] \simeq 10 \mathrm{TeV}
\end{aligned}
$$

Using Eqs. (28) and (36),

$$
M \simeq \frac{\left(M_{*} L_{c}\right) M_{*}}{\sqrt{M_{*} a}} \exp \left[-(a L)^{2}\right]
$$




\begin{tabular}{|l|l|l|}
\hline "Anarchy" type & Improvement 1 & Improvement 2 \\
\hline$\tilde{m}_{Q_{3}, \bar{U}_{3}} \simeq 100 \mathrm{GeV}$ & $\tilde{m}_{Q_{3}, \bar{U}_{3}} \simeq 100 \mathrm{GeV}$ & $\tilde{m}_{Q_{3}, \bar{U}_{3}} \simeq 100 \mathrm{GeV}$ \\
& & $\tilde{m}_{\bar{D}_{1}} \simeq 3.27 \mathrm{TeV}$ \\
$\tilde{m}_{Q_{2}, \bar{U}_{2}, \bar{D}_{1,2,3}} \simeq 13.12 \mathrm{TeV}$ & $\tilde{m}_{Q_{2}, \bar{U}_{2}, \bar{D}_{2,3}} \simeq 13.12 \mathrm{TeV}$ & $\tilde{m}_{Q_{2}, \bar{U}_{2}} \simeq 13.12 \mathrm{TeV}$ \\
$\tilde{m}_{Q_{1}, \bar{U}_{1}} \simeq 10 \mathrm{TeV}$ & $\tilde{m}_{Q_{1}, \bar{U}_{1}, \bar{D}_{1}} \simeq 10 \mathrm{TeV}$ & $\tilde{m}_{Q_{1}, \bar{U}_{1}, \bar{D}_{2,3}} \simeq 10 \mathrm{TeV}$ \\
\hline
\end{tabular}

Table 1: Sfermion mass spectra of three models

is obtained, From Eqs. (36) and (37),

$$
a L \simeq 2.22
$$

is obtained. Eq. (38) can be further rewritten by using Eq. (39) as

$$
M \simeq 7.18 \times 10^{-3} \frac{\left(M_{*} L_{c}\right) M_{*}}{\sqrt{M_{*} a}} .
$$

Taking $M_{*} \simeq 10^{18} \mathrm{GeV}, L_{c}^{-1} \simeq a^{-1} \simeq 10^{16} \mathrm{GeV}$, various scales in our theory are obtained,

$$
L^{-1} \simeq 4.50 \times 10^{17} \mathrm{GeV}, M \simeq 7.18 \times 10^{16} \mathrm{GeV}, \sqrt{F} \simeq 10^{11} \mathrm{GeV} .
$$

As mentioned above, $F<M^{2}$ is satisfied. Other sfermion masses are calculated,

$\tilde{m}_{Q_{2}} \simeq \tilde{m}_{\bar{U}_{2}} \simeq \tilde{m}_{D_{1,2,3}} \simeq 10^{-1} \times \frac{10^{22}}{7.18 \times 10^{16}} \exp \left[-(2.221756-1.977883)^{2}\right] \simeq 13.12 \mathrm{TeV}$.

We note that we have an another choice for the decoupling solution requirement, namely, $\tilde{m}_{Q_{2}} \simeq \tilde{m}_{\bar{U}_{2}} \simeq \tilde{m}_{D_{1,2,3}} \simeq 10 \mathrm{TeV}$. In this case, the squark masses of the first generation $Q_{1}$ and $\bar{U}_{1}$ become $3.8 \mathrm{TeV}$, which is smaller than $10 \mathrm{TeV}$. Therefore, this choice is not plausible for the decoupling solution.

As for the sfermion mass spectrum of Improvement I, they are almost same as that of "Anarchy" model except for the right-handed sdown mass because the difference in the quark sector between two configurations Eqs. (3) and (13) is the location of the righthanded down chiral superfield. As for the Improvement II, the differences in the quark sector between the "Anarchy type" and the Improvement II are the locations of $\bar{D}_{1,2,3}$. In particular, since $\bar{D}_{1}$ is localized a little apart from a SUSY breaking brane, the mass of $\bar{D}_{1}$ becomes slightly small. The sfermion masses spectra are summarized in the Table 1 .

We give some comments on the sfermion mass spectrum. First, as seen in Ref. [12], the mass of the right-handed sbottom $\tilde{m}_{\bar{D}_{3}}$ is around $10 \mathrm{TeV}$ for all models, which is 
somewhat large from the viewpoint of the decoupling solution. This means that the large $\tan \beta$ is preferable. Second, the mass of the right-handed sdown $\tilde{m}_{\bar{D}_{1}}$ is somewhat small in Improvement II because the right-handed down chiral superfield $\bar{D}_{1}$ is localized far apart from the SUSY breaking brane compared to the location where other matter chiral superfields of the first and the second generations. Third, since these sfermion mass spectrum is determined at the compactification scale, the third generation sfermion mass squareds might be driven negative at the weak scale through the two-loop renormalization group effects of the heavy first-two generation sfermion masses [22]. To avoid this, we have to add the extra fields with negative SUSY breaking mass squareds [23]. We do not discuss this point in detail in this paper. Finally, the sfermion mass spectrum from the fermion masses for (11) and (20) are not viable as the decoupling solutions because $\tilde{m}_{\bar{D}_{1,2}}$ or $\tilde{m}_{\bar{D}_{2}}$ becomes the order of $100 \mathrm{GeV}$.

Another interesting sfermion spectrum can be obtained if the SUSY breaking brane is put on the same point where the Higgs fields are localized and the vector-like superfields are introduced on the SUSY breaking brane. In "Anarchy" model with small tan $\beta$, for instance, we obtain the squark mass matrices

$$
\begin{aligned}
M_{u}^{2} & =\left(\begin{array}{cc}
\tilde{m}_{Q}^{2}+m_{u}^{2} & m_{u}\left(a_{u}-\mu \cot \beta\right) \\
m_{u}\left(a_{u}-\mu \cot \beta\right) & \tilde{m}_{\bar{U}}^{2}+m_{u}^{2}
\end{array}\right), \\
M_{d}^{2} & =\left(\begin{array}{cc}
\tilde{m}_{Q}^{2}+m_{d}^{2} & m_{d}\left(a_{d}-\mu \tan \beta\right) \\
m_{d}\left(a_{d}-\mu \tan \beta\right) & \tilde{m}_{\bar{D}}^{2}+m_{d}^{2}
\end{array}\right),
\end{aligned}
$$

where

$$
\begin{aligned}
& \tilde{m}_{Q}^{2} \simeq \tilde{m}_{\bar{U}}^{2} \simeq\left(\begin{array}{ccc}
\epsilon^{4} & \epsilon^{3} & \epsilon^{2} \\
\epsilon^{3} & \epsilon^{2} & \epsilon \\
\epsilon^{2} & \epsilon & 1
\end{array}\right) \frac{1}{M_{*} a}\left(\frac{F}{M}\right)^{2}, \tilde{m}_{\bar{D}}^{2} \simeq \epsilon^{2}\left(\begin{array}{ccc}
1 & 1 & 1 \\
1 & 1 & 1 \\
1 & 1 & 1
\end{array}\right) \frac{1}{M_{*} a}\left(\frac{F}{M}\right)^{2}, \\
& a_{u, d} \equiv A_{u, d} / y_{u, d} \simeq \frac{1}{M_{*} a} \frac{F}{M}
\end{aligned}
$$

and $m_{u}, m_{d}$ are the fermion mass matrices in Eqs. (四). Also, we omitted the elements coming from $S U(2)_{L} \times U(1)_{Y}$ D-terms since the discussion below is not changed. We shall discuss the possibility of the realization of the alignment solution? to SUSY flavor problem 25]. In the sfermion martrices $M_{u}^{2}, M_{d}^{2}$, if the off-diagonal elements are smaller than the diagonal ones, we can obtain the alignment solution since both of the sfermion mass squareds and the fermion mass squareds in each diagonal element take the same

\footnotetext{
${ }^{5}$ In order to be preserve the alignment solution against the quantum corrections, an additional mechanism might be needed in our scenario.
} 
form and these can be simultaneously diagonalized. In the above case, the dominant terms in the diagonal elements are $\tilde{m}_{Q}^{2}, \tilde{m}_{\bar{U}}^{2}$ and $\tilde{m}_{\bar{D}}^{2}$. As for the off-diagonal elements, we note that $\tan \beta \simeq \epsilon m_{t} / m_{b} \simeq 10^{-1}$ in the present case. Assuming that the sfermion masses $\tilde{m}_{Q, \bar{U}, \bar{D}}^{2}$ are around $1 \mathrm{TeV}$, we need the constraint from $\tilde{m}_{Q, \bar{U}}^{2}$

$$
\frac{\epsilon^{2}}{\sqrt{M_{*} a}}\left(\frac{F}{M}\right) \simeq 1 \mathrm{TeV} .
$$

This can be rewritten as

$$
a_{u, d} \simeq \frac{10^{7}}{\sqrt{M_{*} a}} \mathrm{GeV} .
$$

Assuming that $\mu \simeq 100 \mathrm{GeV}$,

$$
\mu \tan \beta \simeq 10 \mathrm{GeV}, \mu \cot \beta \simeq 1 \mathrm{TeV}
$$

are obtained. Which term dominates in the off-diagonal elements depends on the value of $M_{*} a$ in (48). If $a_{u, d}$ are dominant, the conditions to obtain the alignment solution are

$$
\tilde{m}_{Q, \bar{U}}^{2}>m_{u} a_{u}, \tilde{m}_{Q, \bar{D}}^{2}>m_{d} a_{d} .
$$

Most stringent constraint coming from the top quark sector is

$$
1 \mathrm{TeV}^{2}>\frac{10^{9}}{\sqrt{M_{*} a}} \mathrm{GeV}^{2} \Rightarrow M_{*} a>10^{6},
$$

which provides a lower bound of the width of the wave functions.

The slepton mass matrix is

$$
M_{e}^{2}=\left(\begin{array}{cc}
\tilde{m}_{L}^{2}+m_{l}^{2} & m_{l}\left(a_{e}-\mu \tan \beta\right) \\
m_{l}\left(a_{e}-\mu \tan \beta\right) & \tilde{m}_{\bar{E}}^{2}+m_{l}^{2}
\end{array}\right),
$$

where

$$
\begin{aligned}
& \tilde{m}_{L}^{2} \simeq\left(\begin{array}{lll}
1 & 1 & 1 \\
1 & 1 & 1 \\
1 & 1 & 1
\end{array}\right) \frac{1}{M_{*} a}\left(\frac{F}{M}\right)^{2}, \tilde{m}_{\bar{E}}^{2} \simeq \epsilon^{2}\left(\begin{array}{ccc}
\epsilon^{4} & \epsilon^{3} & \epsilon^{2} \\
\epsilon^{3} & \epsilon^{2} & \epsilon \\
\epsilon^{2} & \epsilon & 1
\end{array}\right) \frac{1}{M_{*} a}\left(\frac{F}{M}\right)^{2}, \\
& a_{e} \equiv A_{e} / y_{e} \simeq \frac{1}{M_{*} a} \frac{F}{M},
\end{aligned}
$$

and $m_{l}$ is given in (田).

The squark mass matrices with the large $\tan \beta$ case are written by

$$
\tilde{m}_{Q}^{2} \simeq \tilde{m}_{\bar{U}}^{2} \simeq\left(\begin{array}{ccc}
\epsilon^{4} & \epsilon^{3} & \epsilon^{2} \\
\epsilon^{3} & \epsilon^{2} & \epsilon \\
\epsilon^{2} & \epsilon & 1
\end{array}\right) \frac{1}{M_{*} a}\left(\frac{F}{M}\right)^{2}, \tilde{m}_{\bar{D}}^{2} \simeq\left(\begin{array}{ccc}
1 & 1 & 1 \\
1 & 1 & 1 \\
1 & 1 & 1
\end{array}\right) \frac{1}{M_{*} a}\left(\frac{F}{M}\right)^{2},
$$


$a_{u, d}$ are the same as in the small $\tan \beta$ case and $m_{u}$ and $m_{d}$ are the fermion mass matrices in Eqs. (12). We can repeat the discussion on the alignment solution. In the large $\tan \beta$ case. the constraint for the width of the wave functions becomes $M_{*} a>10^{-2}$. This constraint is always satisfied since $a>M_{*}^{-1}$ in the fat brane scenario.

The slepton mass matrix is

$$
\tilde{m}_{L}^{2} \simeq\left(\begin{array}{ccc}
1 & 1 & 1 \\
1 & 1 & 1 \\
1 & 1 & 1
\end{array}\right) \frac{1}{M_{*} a}\left(\frac{F}{M}\right)^{2}, \tilde{m}_{\bar{E}}^{2} \simeq\left(\begin{array}{ccc}
\epsilon^{4} & \epsilon^{3} & \epsilon^{2} \\
\epsilon^{3} & \epsilon^{2} & \epsilon \\
\epsilon^{2} & \epsilon & 1
\end{array}\right) \frac{1}{M_{*} a}\left(\frac{F}{M}\right)^{2},
$$

$a_{e}$ is the same as the small $\tan \beta$ case and $m_{l}$ is given in (12).

It is important to note that which type of the sfermion mass spectrum is realized depends on the relative location between the SUSY breaking brane and the brane where Higgs fields are localized. Thus, our framework provide a unified picture of the mechanism for generating the sfermion masses.

\section{Summary}

We have discussed the fermion masses and the mixings in the fat brane scenario of a five dimensional SUSY theory. In extra dimensions, the information of Yukawa hierarchy is interpreted as that of the location where the matter fields are localized. There have been many configurations consistent with the experimental data, which corresponds to the way to embed the matter fields and Higgs fields in extra dimensions. We have considered the case in this paper that the matter fields live in the bulk and Higgs fields are localized on the brane. We have found various types of the matter configurations which yields the mass matrices consistent with the fermion mass hierarchy and the flavor mixings. The configurations found in this paper are very simple compared to others in the literature

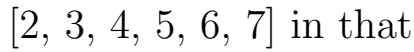

1. The configurations are obtained in a five dimensional theory,

2. The width of the wave functions is common to all the matter fields,

3. The distribution of the matter superfields is very simple.

We have also discussed three types of the sfermion spectrum in our framework. If SUSY breaking brane is located far apart from the region where the matter fields are localized, the sfermion mass spectrum is similar to the gaugino mediation scenario [24]. 
The degeneracy solution to SUSY flavor problem is expected. If SUSY breaking brane is located between the points where the first and the second generations are localized, the decoupling spectrum proposed in [12 is realized. If the SUSY breaking brane is located at the same point where Higgs fields are localized, the sfermion masses are proportional to Yukawa couplings. This case might suggests the alignment solution [25] to SUSY flavor problem. We emphasize that our framework provides a unified picture of the mechanism for generating the sfermion masses. Which scenario is realized depends on the relative location of the SUSY breaking brane and the brane where Higgs fields are localized.

As a future direction, it is interesting to study in detail the slepton mass spectrum and their mixings in our framework.

\section{Acknowledgments}

N.H. is supported by the Grant-in-Aid for Scientific Research, Ministry of Education, Science and Culture, Japan (No.12740146) and N.M. is supported by the Japan Society for the Promotion of Science for Young Scientists (No.08557).

\section{References}

[1] N. Arkani-Hamed and M. Schmaltz, Phys. Rev. D61 (2000) 033005 hep-ph/9903417.

[2] E.A. Mirabelli and M. Schmaltz, Phys. Rev. D61 (2000) 113011 [hep-ph/9912265.

[3] D.E. Kaplan and T.M.P. Tait, JHEP 0006 (2000) 020 hep-ph/0004200].

[4] G.C. Branco, A. de Gouvea and M.N. Rebelo, Phys. Lett. B506 (2001) 115 hep$\mathrm{ph} / 0012289$.

[5] G. Barenboim, G.C. Branco, A. de Gouvea and M.N. Rebelo, Phys. Rev. D64 (2001) 073005 hep-ph/0104312.

[6] D.E. Kaplan and T.M.P. Tait, hep-ph/0110126.

[7] M. Kakizaki and M. Yamaguchi, hep-ph/0110266. 
[8] M. Kakizaki and M. Yamaguchi, Prog. Theor. Phys. (2001) hep-ph/0104103.

[9] N. Maru, Phys. Lett. B522 (2001) 117 hep-ph/0108002].

[10] N. Haba and N. Maru, Phys. Lett. B532 (2002) 93 hep-ph/0201216.

[11] N. Maru, N. Sakai, Y. Sakamura and R. Sugisaka, Nucl. Phys. B616 (2001) 47 [hep-th/0107204].

[12] N. Haba and N. Maru, hep-ph/0202196.

[13] N. Haba, Y. Shimizu, T. Suzuki and K. Ukai, hep-ph/0107190.

[14] N. Haba, T. Kondo and Y. Shimizu, hep-ph/0202191.

[15] T. Yanagida, in Proceedings of the Workshop on Unified Theory and Baryon Number of the Universe, eds. O. Sawada and A. Sugamoto, KEK report 79-18, 1979; M. GellMann, P. Ramond and R. Slansky, in Supergravity, eds. P. van Nieuwenhuizen and D. Freedman, North Holland, Amsterdam, 1979.

[16] N. Cabibbo, Phys. Rev. Lett. 10 (1963) 531; M. Kobayashi and T. Maskawa, Prog. Theor. Phys. 49 (1973) 652.

[17] Z. Maki, M. Nakagawa and S. Sakata, Prog. Theor. Phys. 28 (1962) 870.

[18] K.S. Babu and S.M. Barr, Phys. Lett. B381 (1996) 202 hep-ph/9511446. S.M. Barr, Phys. Rev. D55 (1997) 1659 [hep-ph/9607419].

[19] M.J. Strassler, Phys. Lett. B376 (1996) 119 hep-ph/9510342]; N. Haba, Phys. Rev. D59 (1999) 035011 hep-ph/9807552; K. Yoshioka, Mod. Phys. Lett. A15 (2000) 29 hep-ph/9904433]; J. Hisano, K. Kurosawa and Y. Nomura, Nucl. Phys. B584 (2000) 3 hep-ph/0002286; T. Blazek and S.F. King, Phys. Lett. B518 (2001) 109 [hep-ph/0105005.

[20] N. Haba and H. Murayama, Phys. Rev. D63 (2001) 053010 hep-ph/0009174].

[21] R. Rosenfeld and J. L. Rosner, Phys. Lett. B516 (2001) 408[hep-ph/0106335].

[22] N. Arkani-Hamed and H. Murayama, Phys. Rev. D56 (1997) 6733 hep-ph/9703259;

K. Agashe and M. Graesser, Phys. Rev. D59 (1999) 015007 [hep-ph/9801446. 
[23] J. Hisano, K. Kurosawa and Y. Nomura, Nucl. Phys. B584 (2000) 3 hep$\mathrm{ph} / 0002286]$.

[24] D.E. Kaplan, G.D. Kribs and M. Schmaltz, Phys. Rev. D62 (2000) 035010 hepph/9911293]; Z. Chacko, M.A. Luty, A.E. Nelson and E. Ponton, JHEP 0001 (2000) 003 hep-ph/9911323.

[25] Y. Nir and N. Seiberg, Phys. Lett. B309 (1993) 337 hep-ph/9304307. 